\title{
Light-cone behavior of the pion Bethe-Salpeter wave function in the ladder model*
}

\author{
Marvin L. Goldberger and Davison E. Soper \\ Joseph Henry Laboratories, Princeton University, Princeton, New Jersey 08540 \\ Alan H. Guth \\ Department of Physics, Columbia University, New York, New York 10027 \\ (Received 21 July 1976)
}

\begin{abstract}
The Bethe-Salpeter wave function $\chi\left(q^{\nu}+P^{\nu}, q^{\nu}\right)$ for two spin-1/2 quarks bound by the exchange of a scalar meson is examined in the ladder model. We seek the behavior of $\chi$ as the squared momentum, $(q+P)^{2}$, on one leg becomes infinite while the squared momentum, $q^{2}$, on the other leg remains fixed. This behavior is investigated by making a Wick rotation, expanding $\chi$ in partial-wave amplitudes $\chi_{J}^{i}\left(q^{2}\right)$ of the group $\mathrm{O}(4)$, and then looking for the rightmost poles of $\chi_{J}^{i}\left(q^{2}\right)$ in the complex $J$ plane. Our results verify (in the ladder model) the useful hypothesis that the locations of these poles are independent of $q^{2}$ and can thus be computed in the $q^{2} \rightarrow \infty$ limit by using conformal invariance.
\end{abstract}

\section{INTRODUCTION}

In this paper we study the Bethe-Salpeter boundstate wave function for a pion, considered as a bound state of two spin- $\frac{1}{2}$ quarks:

$$
\chi_{\alpha \beta}\left(q^{\nu}+P^{\nu}, q^{\nu}\right)=\int d x e^{i q \cdot x}\left\langle 0\left|T\left\{\psi_{\alpha}^{(A)}(0) \bar{\psi}_{\beta}^{B}(x)\right\}\right| P^{\nu}\right\rangle
$$

(see Fig. 1). We adopt the Bethe-Salpeter ladder model with scalar-meson exchange as a model for the binding interaction between the quarks (see Sec. II). The ladder-model kernel is not expected to be a quantitatively close approximation to the full Bethe-Salpeter kernel in a $g \bar{\psi} \psi \phi$ quantum field theory. However, we are interested only in the qualitative features of the wave function in certain large-momentum limits. For this purpose the ladder-model integral operator is a useful replacement for the full integral operator in the Bethe-Salpeter equation because (1) it is simple, and (2) it shares with the full integral operator the property of having physical (=canonical + anomalous) dimension exactly zero in the largemomentum limit. ${ }^{1}$ Thus one can reasonably hope that the ladder model gives qualitatively correct. information about large-momentum limits of the wave function in a $g \bar{\psi} \psi \phi$ theory and, more important, that by studying the ladder model one can

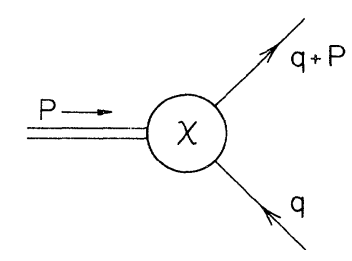

FIG. 1. The Bethe-Salpeter wave function. glean some hints about how to extract the desired limits from the full Bethe-Salpeter equation. (One might even hope to learn something about asymptotically free gauge theories, which are likely to prove more relevant to the real world than scalarexchange theories, but we restrict ourselves here to the scalar-exchange theory in order to avoid problems associated with gauge invariance.) We seek two pieces of information about $\chi\left(q^{\nu}+P^{\nu}, q^{\nu}\right)$. First, we wish to know how $\chi$ behaves in the "short-distance" limit $q^{2} \rightarrow \infty,(q+P)^{2} \rightarrow \infty, q^{2} /$ $(q+P)^{2}=1$. This short-distance limit was treated in some detail in a ladder model by two of the present author $\mathrm{s}^{2}$ and in model-independent analyses by Callan and Gross ${ }^{3}$ (using the renormalization group) and by the present author $\mathrm{s}^{4}$ (using the operator-product expansion). Thus we will give only a superficial treatment of this limit here. The second, and much more elusive, piece of information that we seek is the behavior of $\chi$ as $(q+P)^{2}-\infty$ with $q^{2}$ fixed. This information is important, for instance, in determining the behavior of the pion form factor $F\left(Q^{2}\right)$ for large $Q^{2} .^{3,4,5}$ The behavior of $\chi$ when $(q+P)^{2}$ and $q^{2}$ are both large compared to the mass scale of the theory but $(q+P)^{2} \gg q^{2}$ can be determined by using conformal invariance without resorting to the ladder model (see Callan and Gross, ${ }^{3}$ Menotti $^{5}$ and, for a more detailed treatment, Ref. 4). However, conformal-invariance arguments do not apply when $q^{2}$ is not large. When faced with this difficulty in a calculation of, say, the pion form factor, ${ }^{3,4,5}$ one has been forced to adopt the conformal-invariance result even for finite $q^{2}$ and hope that the dependence of $\chi$ on $(q+P)^{2}$ is the same for finite $q^{2}$ as it is for large $q^{2}$. Our purpose here is to show that this hope is justified, at least in the ladder model.

The limit $(q+P)^{2} \rightarrow \infty$ with $q^{2}$ fixed is difficult to 
attack directly, even in the ladder model. The reason for the difficulty is that in this limit the vector $q^{\mu}$ must be large and point very nearly along the light cone. However, one does not have very good control over the Bethe-Salpeter kernel when the momenta involved are nearly lightlike. Accordingly, it is found helpful to first make a Wick rotation of the momentum $q^{\mu}$ and the loop momentum in the Bethe-Salpeter equation. In the Wick-rotated, Euclidean world the momentum integration is well behaved. It is, of course, impossible to take the limit $q \cdot P \rightarrow \infty$ with $q^{2}$ and $P^{2}\left(=-M^{2}\right)$ fixed while remaining in the Euclidean world. However, one can learn about this limit by expanding $\chi$ in certain functions $G_{j}^{i}$ of $\cos \theta=\hat{q} \cdot \hat{P}$ (see Sec. II):

$$
\chi\left(q^{\nu}+P^{\nu}, q^{\nu}\right)=\sum_{J=0}^{\infty} \sum_{i=1}^{4} \chi_{J}^{i}\left(q^{2}\right) G_{J}^{i}\left(\hat{q}^{\nu}, \hat{P}^{\nu}\right) .
$$

(These functions are appropriate representation functions of the rotation group in the four-dimensional Euclidean space.) One can analytically continue the Bethe-n alpeter equation into the complex $J$ plane and thereby locate (Sec. III) the rightmost poles in the $J$ plane of the partial-wave amplitudes $\chi_{J}^{i}\left(q^{2}\right)$. These poles determine the behavior of $\chi\left(q^{\nu}+P^{\nu}, q^{\nu}\right)$ when $\cos \theta=\left[(q+P)^{2}-q^{2}-P^{2}\right] /$ $(2 i M q) \rightarrow \infty$ with $q^{2}$ fixed, as one sees by performing a Sommerfeld-Watson transformation on the sum, as explained in Appendix A.

One should perhaps note that this translation from the variables $J, q^{2}$ back to the momentum variables $(q+P)^{2}, q^{2}$ is not necessary for the pion form-factor calculation of Ref. 4 . In that calculation one also avoids evaluation of a Minkowski loop integration with large, nearly lightlike momenta by making a Wick rotation and expanding in $O(4)$ eigenfunctions. The $J$-plane poles of the pion partial-wave amplitudes then emerge as divectly relevant to the asymptotic behavior of the pion form factor.

The paper is organized as follows. In Sec. II we introduce the $O(4)$ expansion of the wave function and rewrite the ladder-model Bethe-Salpeter equation using the variables $J, q^{2}$. In Sec. III we use the Bethe-Salpeter equation to examine the behavior of the partial-wave amplitudes for large $q^{2}$, determining both the powers of $q^{2}$ that occur and the locations $J_{\alpha}(\infty)$ of the $J$-plane poles in the $q^{2} \rightarrow \infty$ limit. In Sec. IV we turn to the more difficult problem of determining the $J$-plane structure of $\chi_{J}^{i}\left(q^{2}\right)$ for finite $q^{2}$. We find that $\chi_{J}^{i}\left(q^{2}\right)$ still has poles for finite $q^{2}$ and that the positions $J_{\alpha}\left(q^{2}\right)$ of these poles are independent of $q^{2}$ and are in fact the same as the pole locations $J_{\alpha}(\infty)$ found in the $q^{2} \rightarrow \infty$ limit. We also find that the residues associated with these poles have a simple property that is needed in the form-factor calculation of Ref. 4. These conclusions are stated more fully in Sec. V. The connection between $J$-plane poles and large- $(q+P)^{2}$ behavior, via the SommerfeldWatson transformation, is explained in Appendix A. Certain technical details of the problem of locating poles are explained in Appendix B.

\section{THE LADDER EQUATION AND ITS O(4) REDUCTION}

We consider a model in which the "pion" is a mass- $M$ bound state of a spin- $\frac{1}{2}$ quark of type $A$ and a spin- $\frac{1}{2}$ antiquark of type $\bar{B}$. The object to be investigated is the Bethe-Salpeter wave function $^{6}$

$\chi_{\alpha \beta}\left(q^{\nu}+P^{\nu}, q^{\nu}\right)=\int d x e^{i q_{\circ} x}\left\langle 0\left|T\left\{\psi_{\alpha}^{(A)}(0) \bar{\psi}_{\beta}^{(B)}(x)\right\}\right| P^{\nu}\right\rangle$.

Here $\left|P^{\nu}\right\rangle$ is the one-pion state, with $P^{2}=-M^{2}$. (Our notation ${ }^{7}$ is that of Ref. 4.)

The wave function obeys the Bethe-Salpeter equation illustrated in Fig. 2, where $S^{-1}$ is the full quark inverse propagator and $K$ is the full two-particle-irreducible four-quark amputated Green's function in, say, a $g \bar{\psi} \psi \phi$ field theory. Of course, we are unable to deal with the full equation, so we adopt the ladder approximation in which $S^{-1}$ and $K$ are replaced by their lowestorder forms in perturbation theory. The resulting equation is

$$
\begin{aligned}
(i q q+i \not p+m) \chi\left(q^{\nu}\right. & \left.+P^{\nu}, q^{\nu}\right)(i \not q+m) \\
& =g^{2} \int \frac{d^{4} k}{(2 \pi)^{4}} \frac{1}{(q-k)^{2}+\mu^{2}} \chi\left(k^{\nu}+P^{\nu}, k^{\nu}\right)
\end{aligned}
$$

as illustrated in Fig. 3. Here the vectors $q^{\nu}$ and $k^{\nu}$ have been Wick-rotated ${ }^{8}$ to Euclidean values $q^{4} \equiv i q^{0}$ and $k^{4} \equiv i k^{0}$ real. The vector $P^{\mu}$ has not been Wick-rotated since we must keep $P^{2}=-M^{2}$. Thus we take $P^{4}=i M, P^{1}=P^{2}=P^{3}=0$.

Let us expand $\chi$ in representation functions of the four-dimensional rotation group, $\mathrm{O}(4)$, as ex-

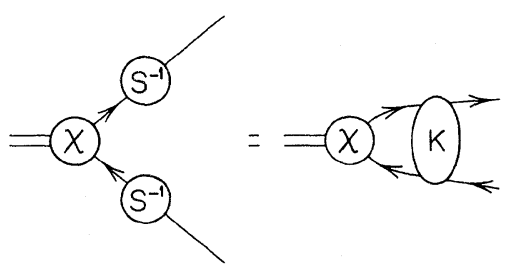

FIG. 2. The full Bethe-Salpeter equation. 

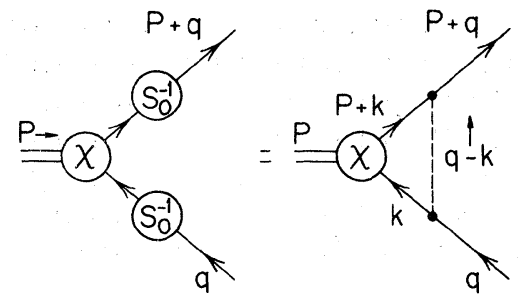

FIG. 3. The ladder-model Bethe-Salpeter equation.

plained in Refs. 4 and 2:

$$
\chi\left(q^{\nu}+P^{\nu}, q^{\nu}\right)_{\alpha \beta}=\sum_{J=0}^{\infty} \sum_{i=1}^{4} \chi_{J}^{i}\left(q^{2}\right) G_{J}^{i}\left(\hat{q}^{\nu}\right)_{\alpha \beta} .
$$

The functions $G_{J}^{i}$ are

$$
\begin{aligned}
G_{J}^{1}= & i \gamma_{5}(-2 i M)^{J}\left\{\hat{q}^{\mu_{1}} \cdots \hat{q}^{\mu_{J}}\right\}\left\{\hat{P}_{\mu_{1}} \cdots \hat{P}_{\mu_{J}}\right\} \\
= & i \gamma_{5}(-i M)^{J} U_{J}(\hat{P} \cdot \hat{q}), \\
G_{J}^{2}= & -\frac{1}{2} i\left[\gamma_{\alpha}, \gamma^{\beta}\right] \gamma_{5}(-2 i M)^{J}\left\{\hat{q}^{\mu_{1}} \cdots \hat{q}^{\mu_{J-1}} \hat{q}^{\alpha}\right\} \\
& \times\left\{\hat{P}_{\mu_{1}} \cdots \hat{P}_{\mu_{J-1}} \hat{P}_{\beta}\right\} \\
= & -\frac{1}{2} i[\hat{q}, \hat{P}] \gamma_{5}(-i M)^{J} \frac{1}{J} U_{J}^{\prime}(\hat{P} \cdot \hat{q}), \\
G_{J}^{3}= & \gamma^{\alpha} \gamma_{5}(-2 i M)^{J}\left\{\hat{q}_{1}^{\mu_{1}} \cdots \hat{q}_{J-1}^{\mu_{J}}\right\}\left\{\hat{P}_{\mu_{1}} \cdots \hat{P}_{\mu_{J-1}} \hat{P}_{\alpha}\right\} \\
= & (-i M)^{J} \frac{1}{J}\left[\hat{p}_{\gamma_{5}} U_{J}^{\prime}(\hat{P} \cdot \hat{q})-\hat{q} \gamma_{5} U_{J-1}^{\prime}(\hat{P} \cdot \hat{q})\right], \\
G_{J}^{4}= & -2 \gamma_{\alpha} \gamma_{5}(-2 i M)^{J}\left\{\hat{q}^{\mu_{1}} \cdots \hat{q}^{\mu} \hat{q}^{\alpha}\right\}\left\{\hat{P}_{\mu_{1}} \cdots \hat{P}_{\mu_{J}}\right\} \\
= & -(-i M)^{J} \frac{1}{J+1}\left[\hat{q} \gamma_{5} U_{J+1}^{\prime}(\hat{P} \cdot \hat{q})-\hat{P}_{\gamma_{5}} U_{J}^{\prime}(\hat{P} \cdot \hat{q})\right] .
\end{aligned}
$$

Here $\hat{q}^{\nu}=q^{\nu} /\left(q^{2}\right)^{1 / 2}$ and $\hat{P}^{\nu}=\left(\hat{P}^{1}, \hat{P}^{2}, \hat{P}^{3}, \hat{P}^{4}\right)$ $=(0,0,0,1) \cdot{ }^{17}$ The braces \{\} indicate that the traceless symmetric part of the tensor inside is to be taken. ${ }^{9}$ The function $U_{J}(\cos \theta)$ is the Chebyshev polynomial ${ }^{10}$

$$
U_{J}(\cos \theta)=\frac{\sin [(J+1) \theta]}{\sin \theta}
$$

and $U_{J}^{\prime}=d U_{J} / d \cos \theta$. As explained in Ref. 2, these functions are eigenfunctions of the $O(4)$ Casimir operators (acting on $q^{\nu}$ and the spinor indices) with eigenvalues $\left(J_{1}, J_{2}\right)=\left(\frac{1}{2} J, \frac{1}{2} J\right)$.

It is a straightforward exercise to transform the Bethe-Salpeter (BS) equation to the $\mathrm{O}(4)$ angular momentum representation by inserting the $O(4)$ expansion of the wave functions into the momentum-space BS equation and matching the coefficients of $G_{J}^{i}(\hat{q})$ on both sides of the equation.

For the left-hand side of the BS equation one needs to work out the matrices $a_{J}, b_{J}, c_{J}$ defined by

$$
\begin{aligned}
(i \not q & +i \not p+m) G_{J}^{l}(\hat{q})(i q q+m) \\
& =\sum_{k=1}^{4}\left[G_{J=1}^{k}(\hat{q}) a_{J}^{k l}\left(q^{2}\right)+G_{J}^{k}(\hat{q}) b_{J}^{k l}\left(q^{2}\right)+G_{J+1}^{k}(\hat{q}) c_{J}^{k l}\left(q^{2}\right)\right] .
\end{aligned}
$$

Notice that only G's with angular momentum $J_{-} 1$, $J$, and $J+1$ appear here, since the inverse propagators carry only one unit of angular momentum, that is, one factor of $P^{\mu}$. This is a special feature of the ladder model.

For the right-hand side of the BS equation one needs to carry out the angular integration. The basic integral for this purpose is ${ }^{11}$

$$
\begin{aligned}
(2 \pi)^{-4} \int d \Omega_{\hat{k}} \frac{\left\{\hat{k}^{\mu_{1}} \cdots \cdot \hat{k}^{\mu} L\right\}}{(q-k)^{2}+\mu^{2}} & \\
= & \frac{1}{8 \pi^{2} q k(L+1)} e^{-(L+1) \omega}\left\{\hat{q}^{\mu_{1}} \cdots \hat{q}^{\mu} L\right\},
\end{aligned}
$$

where $\omega \equiv \omega(q, k)$ is defined by

$$
\cosh [\omega(q, k)]=\frac{q^{2}+k^{2}+\omega^{2}}{2 q k}, \omega>0 .
$$

Thus the functions $G_{J}^{i}$ are eigenfunctions of the angular-integral operator with eigenvalues determined by the number $L$ of $q$ 's in $G_{J}^{i}(\hat{q})\left(L=J+\Delta_{i}\right.$ with $\left.\Delta_{1}=\Delta_{2}=0, \Delta_{3}=-1, \Delta_{4}=+1\right)$. Having done the angular integration, we are left with an integral operator that acts on the partial-wave amplitudes $\chi_{J}^{i}\left(k^{2}\right)$. Let us call this integral operator $T_{L}$ :

$$
\left[T_{L} h\right]\left(q^{2}\right) \equiv \frac{(g / 2 \pi)^{2}}{2(L+1)} \int_{0}^{\infty} d k \frac{k^{2}}{q} e^{-(L+1) \omega} h\left(k^{2}\right) .
$$

The resulting BS equation for the partial-wave amplitudes is

$$
0=\sum_{l=1}^{4}\left(A_{J+1}^{j l} \chi_{J+1}^{l}+B_{J}^{j l} \chi_{J}^{l}+C_{J=1}^{j l} \chi_{J=1}^{l}\right),
$$

where

$$
A_{J}=\left[\begin{array}{cccc}
\frac{1}{2} M^{2} q & \frac{1}{2} \frac{J+2}{J} M^{2} q & \frac{J+1}{J} M^{2} m & 0 \\
-\frac{1}{2} \frac{J-1}{J+1} M^{2} q & -\frac{1}{2} \frac{(J-1)(J+2)}{J(J+1)} M^{2} q & -\frac{J-1}{J} M^{2} m & 0 \\
0 & 0 & 0 & 0 \\
\frac{1}{2} \frac{J}{J+1} M^{2} m & \frac{1}{2} \frac{J+2}{J+1} M^{2} m & -M^{2} q & 0
\end{array}\right],
$$




$$
\begin{aligned}
& B_{J}=\left[\begin{array}{cccc}
m^{2}+q^{2}-T_{J} & 0 & 0 & 0 \\
0 & m^{2}-q^{2}-T_{J} & -2 m q & -2 \frac{J}{J+1} m q \\
0 & \frac{J+2}{J+1} m q & m^{2}-\frac{q^{2}}{J+1}-T_{J=1} & -\frac{J(J+2)}{(J+1)^{2}} q^{2} \\
0 & m q & -q^{2} & m^{2}+\frac{q^{2}}{J+1}-T_{J+1}
\end{array}\right], \\
& C_{J}=\left[\begin{array}{cccc}
-\frac{1}{2} q & \frac{1}{2} q & 0 & m \\
-\frac{1}{2} q & \frac{1}{2} q & 0 & m \\
\frac{1}{2} m & -\frac{1}{2} m & 0 & q \\
0 & 0 & 0 & 0
\end{array}\right]
\end{aligned}
$$

Notice that the integral operators $T_{L}$ have been incorporated into the matrix $B_{J} ; T_{L} f$ means $\left[T_{L} f\right]\left(q^{2}\right)$ as specified in Eq. (2.7)。

\section{SOLUTION FOR LARGE $q^{2}$}

The large- $q^{2}$ behavior of $\chi_{j}^{i}\left(q^{2}\right)$ can be investigated by a simple heuristic method if one is willing to accept as an ansatz certain information about the general structure of $\chi_{J}^{i}\left(q^{2}\right)$ that is obtained from a more thorough analysis. ${ }^{2}$ This analysis shows that $\chi_{J}^{i}\left(q^{2}\right)$ is a linear combination of certain elementary amplitudes $\chi_{j}^{i}\left(q^{2}, \alpha\right)$ labelled by an index $\alpha$ :

$$
\chi_{J}^{i}\left(q^{2}\right) \sim \sum_{\alpha} c(\alpha) \chi_{J}^{i}\left(q^{2}, \alpha\right) .
$$

The elementary amplitudes have the form

$$
\chi_{J}^{i}\left(q^{2}, \alpha\right) \sim \sum_{N=0} \sum_{l=0}^{N} a_{J}^{i}(\alpha, N, l)(\ln q)^{l} q^{-3-s(\alpha)-J-N} .
$$

The " " in these equations indicates equality term by term in an asymptotic expansion about $q^{2}=\infty$. Each of the elementary amplitudes is an independent solution of the BS equation order by order in the large $-q^{2}$ expansion. The power $s(\alpha)$ is determined by a consistency condition, as we will see. The coefficients $a_{J}^{i}(\alpha, N, l)$ for a given $N=N_{0}$ and $J=J_{0}$ are determined by the coefficients for $N \leq N_{0}, 2 J+N \leq 2 J_{0}+N_{0}$. Thus any given coefficient can be computed (in terms of a starting coefficient) with a finite amount of computation; we will compute the coefficients $a_{J}^{i}(\alpha, 0,0)$ in closed form below. The large $-q^{2}$ analysis determines the elementary amplitudes to any desired order, but does not determine what linear combination of the elementary amplitudes occurs in the complete wave function $\chi_{J}^{i}\left(q^{2}\right)$. The required coefficients $c(\alpha)$ in Eq. (3.1), as well as the bound-state mass $M$, are presumably fixed by demanding suitable infrared behavior of $\chi_{J}^{i}\left(q^{2}\right)$.

We will be interested only in the leading behavior of each elementary amplitude as $q^{2}-\infty$ with $J$ fixed, that is, the $N=0$ term in Eq. (3.2). This leading part of $\chi_{J}^{i}\left(\alpha, q^{2}\right)$ can be obtained by inserting the ansatz

$$
\chi_{J}^{i}\left(q^{2}\right)=a_{J}^{i} q^{-3-s-J}
$$

into the BS equation and demanding that the equation be satisfied to leading order in $q^{2}$ (that is, to order $\left.q^{-1-s-J}\right)$.

When the integral operator $T_{L}$ is applied to the function $f(k)=k^{-3-s-J}$, the leading behavior of the resulting function is

$$
\begin{gathered}
{\left[T_{L} f\right]\left(q^{2}\right) \sim \frac{(g / 2 \pi)^{2}}{2(L+1)} \int_{0}^{\infty} d k \frac{k^{2}}{q}\left[\min \left(\frac{k}{q}, \frac{q}{k}\right)\right]^{L+1} f\left(k^{2}\right)} \\
=\frac{(g / 2 \pi)^{2}}{(L+1)^{2}-(J+s)^{2}} q^{-1 m s-J} .
\end{gathered}
$$

(A subtle point connected with this integral is explained toward the end of Appendix B.)

We now insert the ansatz (3.3) for $\chi_{J}^{i}\left(q^{2}\right)$ into the BS equation (2.8) and use the result (3.4) for $T_{L} \chi$. The leading terms are of order $q^{-1-s-J}$; we demand that the coefficient of $q^{-1-s-J}$ vanish. (We are not concerned with the coefficients of nonleading powers of $q$ since we wish to determine only the leading terms in the wave function.) Several terms of the matrices $B_{J}$ and $C_{J-1}$ can therefore be neglected, as can the entire matrix $A_{J+1}$. The result is

$$
0=\sum_{l=1}^{4}\left(\hat{B}_{J}^{j l} a_{J}^{l}+\hat{C}_{J=1}^{j l} a_{J=1}^{l}\right)
$$

The matrices $\hat{B}_{J}$ and $\hat{C}_{J}$ have a block diagonal form 


$$
\hat{B}_{J}=\left(\begin{array}{cc}
b_{+, J} & 0 \\
0 & b_{-, J}
\end{array}\right), \hat{C}_{J=1}=\left(\begin{array}{cc}
c_{+} & 0 \\
0 & c_{-}
\end{array}\right),
$$

where

$$
\begin{aligned}
& b_{+, J}=\left(\begin{array}{cc}
1-\frac{(g / 2 \pi)^{2}}{(J+1)^{2}-(J+s)^{2}} & 0 \\
0 & -1-\frac{(g / 2 \pi)^{2}}{(J+1)^{2}-(J+s)^{2}}
\end{array}\right), \\
& b_{-, J}=\left(\begin{array}{cc}
-\frac{1}{J+1}-\frac{(g / 2 \pi)^{2}}{J^{2}-(J+s)^{2}} & -\frac{J(J+2)}{(J+1)^{2}} \\
-1 & \frac{1}{J+1}-\frac{(g / 2 \pi)^{2}}{(J+2)^{2}-(J+s)^{2}}
\end{array}\right), \\
& c_{+}=\left(\begin{array}{cc}
-\frac{1}{2} & +\frac{1}{2} \\
-\frac{1}{2} & +\frac{1}{2}
\end{array}\right), \\
& c_{-}=\left(\begin{array}{ll}
0 & 1 \\
0 & 0
\end{array}\right) .
\end{aligned}
$$

\section{A. Eigenvalue condition for the exponent $s$}

The large $-q^{2}$ BS equation has been derived for $J=2,3, \ldots$ For $J=0$ and $J=1$ there is a technical difficulty due to the fact that there are no basis functions $G_{J}^{i}$ for $J=-1$ and also no basis functions $G_{J}^{2}, G_{J}^{3}$ for $J=0$. Thus the partial-wave amplitudes $\chi_{-1}^{i}, \chi_{0}^{2}$, and $\chi_{0}^{3}$ are as yet undefined. Apparently, the BS equation (2.8) and its large- $q^{2}$ form (3.5) remain true for $J=0$ and $J=1$ if we define $\chi_{-1}^{i}=\chi_{0}^{2}$ $=\chi_{0}^{3}=0$ and thus also $a_{-1}^{i}=a_{0}^{2}=a_{0}^{3}=0$.

Since $a_{-1}=0$, Eq. (3.5) for $J=0$ implies that either $a_{0}=0$ or $B_{0} a_{0}=0$. In the case $a_{0}=0$ the equation for $J=1$ implies that either $a_{1}=0$ or $B_{1} a_{1}=0$. Continuing this argument, we conclude that there must be some integer $j_{0}$ such that $a_{-1}=a_{0}=\cdots$ $=a_{J_{0}-1}=0 \mathrm{but}$

$$
\begin{aligned}
& a_{J_{0}}^{l} \neq 0, \\
& B_{J_{0}}^{j l} a_{J_{0}}^{l}=0 .
\end{aligned}
$$

The eigenvalue condition (3.7) implies that the exponent $s$ must be chosen so that the determinant of $\hat{B}_{J_{0}}$ vanishes. As we will see, there are four solutions for $s$, and four corresponding independent solutions of the large $-q^{2}$ BS equation, for each choice of $J_{0}$.

\section{B. Solution of the large- $q^{2}$ BS equation}

The large $-q^{2}$ BS equation (3.5) is a rather simple linear recursion equation and can be easily solved in closed form. To solve it, one first notes that because of the block-diagonal form of $\hat{B}_{J}$ and $\hat{C}_{J-1}$, the equation breaks up into two independent equations, one for the two-dimensional vector $\left(a_{J}^{1}, a_{J}^{2}\right)$ and one for the two-dimensional vector $\left(a_{J}^{3}, a_{J}^{4}\right)$. (Chiral invariance as $q^{2} \rightarrow \infty$ is responsible for this simplification.) Each two-dimensional equation can be further simplified by noting that the matrices $c_{ \pm}$are singular. Thus, for instance, the two-dimensional vector $\left(a_{J}^{1}, a_{J}^{2}\right) \equiv a_{J}^{(+)}=-\left(b_{+, J}\right)^{-1} c_{+} a_{J=1}^{(+)}$ must lie in a certain one-dimensional subspace:

$$
a_{J}^{(+)}=\alpha_{J}\left(\begin{array}{c}
(J+1)^{2}-(J+s)^{2}+(g / 2 \pi)^{2} \\
-(J+1)^{2}+(J+s)^{2}+(g / 2 \pi)^{2}
\end{array}\right) .
$$

The coefficients $\alpha_{J}$ are then seen to obey a certain (one-dimensional) linear recursion equation:

$$
\alpha_{J}=\frac{\left[2 J(1-s)+1-s^{2}\right]\left[2 J(1-s)-(1-s)^{2}\right]}{\left[2 J(1-s)+1-s^{2}-(g / 2 \pi)^{2}\right]\left[2 J(1-s)+1-s^{2}+(g / 2 \pi)^{2}\right]} \alpha_{J-1} .
$$

This recursion equation is evidently solved by a combination of four $\Gamma$ functions. The solution of the equation for $\left(a_{J}^{3}, a_{J}^{4}\right)$ proceeds along similar lines.
Let us examine first the solution for $a_{J}^{i}$ with $\left(a_{J}^{1}, a_{J}^{2}\right) \neq 0$. One finds (after choosing a convenient normalization) 


$$
\begin{aligned}
& a_{J}^{l}=\frac{\Gamma\left(J+\frac{1}{2} s+\frac{3}{2}\right) \Gamma\left(J+\frac{1}{2} s+\frac{1}{2}\right)}{\Gamma\left(J+\frac{1}{2} s+\frac{3}{2}-(g / 2 \pi)^{2} / 2(1-s)\right) \Gamma\left(J+\frac{1}{2} s+\frac{1}{2}+(g / 2 \pi)^{2} / 2(1-s)\right)}, \\
& a_{J}^{2}=-\frac{\Gamma\left(J+\frac{1}{2} s+\frac{3}{2}\right) \Gamma\left(J+\frac{1}{2} s+\frac{1}{2}\right)}{\Gamma\left(J+\frac{1}{2} s+\frac{1}{2}-(g / 2 \pi)^{2} / 2(1-s)\right) \Gamma\left(J+\frac{1}{2} s+\frac{3}{2}+(g / 2 \pi)^{2} / 2(1-s)\right)}, \\
& a_{J}^{3}=0 \\
& a_{J}^{4}=0 .
\end{aligned}
$$

The value of the exponent $s$ can be chosen so that $a_{J_{0}-1}^{i}=a_{J_{0-2}}^{i}=\cdots=0$ by choosing $s$ such that

$$
J_{0}+\frac{1}{2} s+\frac{1}{2}-(g / 2 \pi)^{2} / 2(1-s)=0
$$

or

$$
J_{0}+\frac{1}{2} s+\frac{1}{2}+(g / 2 \pi)^{2} / 2(1-s)=0 .
$$

The first choice gives $a_{J_{0}}^{i}=$ const $\times(1,0,0,0)$, while the second choice gives $a_{J_{0}}^{i}=$ const $\times(0,1,0,0)$.

There is no $O(4)$ function $G_{0}^{2}$, so the second choice is physically possible only for $J_{0} \geq 1$, whereas the first choice is possible for $J_{0} \geq 0$. [In addition, charge-conjugation invariance in the case of a $\pi^{0}$ or $G$ parity in the case of a $\pi^{ \pm}$limits $J_{0}$ to the values $0,2,4, \ldots$ in the case of the $(1,0,0,0)$ solution and to the values $J_{0}=1,3,5, \ldots$ in the case of the $(0,1,0,0)$ solution. $\left.{ }^{4}\right]$

Equations (3.9) and (3.10) are quadratic equations for $s$. Each has one root that lies near $s=1$ when $g \ll 1$ :

$$
s=\left[\left(J_{0}+1\right)^{2}-(g / 2 \pi)^{2}\right]^{1 / 2}-J_{0}
$$

for $a_{J_{0}} \propto(1,0,0,0)$ and

$$
s=\left[\left(J_{0}+1\right)^{2}+(g / 2 \pi)^{2}\right]^{1 / 2}-J_{0}
$$

for $a_{J_{0}} \propto(0,1,0,0)$. Each of equations (3.9) and (3.10) also has a root that lies near $s=-1-2 J_{0}$. We reject this root as unphysical since it implies an unacceptably singular large- $q^{2}$ behavior of the BS wave function. ${ }^{2}$ [For instance, the unphysical root leads, using the methods of Ref. 4, to a pion form factor $F\left(Q^{2}\right) \sim\left(Q^{2}\right)^{J_{0}}$, with subtractions required to regularize the form-factor loop integration if $J_{0} \geq 1$.]

We now turn to the solution for $a_{J}^{i}$ with $\left(a_{J}^{3}, a_{J}^{4}\right) \neq 0$. One finds after a certain amount of algebra (and the choice of a convenient normalization)

$$
\begin{aligned}
& a_{J}^{1}=0, \\
& a_{J}^{2}=0, \\
& a_{J}^{3}=\left[\frac{1}{J+1}-\frac{(g / 2 \pi)^{2}}{2(2-s)\left(J+1+\frac{1}{2} s\right)}\right] a_{J}^{4}, \\
& a_{J}^{4}=\frac{\Gamma\left(J+1+\frac{1}{2} s\right) \Gamma\left(J+2+\frac{1}{2} s\right)}{\Gamma\left(J-f_{+}(s)\right) \Gamma\left(J-f_{-}(s)\right)},
\end{aligned}
$$

where

$$
\begin{aligned}
f_{ \pm}(s)= & -\frac{3}{2}-\frac{1}{2} s \\
& \pm \frac{1}{2}\left\{1+\left[(g / 2 \pi)^{2} / s(2-s)\right]\left[4-(g / 2 \pi)^{2}\right]\right]^{1 / 2} .
\end{aligned}
$$

The value of the exponent $s$ is to be chosen so that $a_{J_{0}}^{i} \neq 0 ; a_{J_{0}-1}^{i}=a_{J_{0}-2}^{i}=\cdots=0$, where, for $G$-parity reasons, $J_{0}$ can be $1,3,5, \ldots$ Thus we choose $s$ to be a solution of

$$
f_{ \pm}(s)=J_{0}-1 \text {. }
$$

There are four solutions to this equation, as shown in Fig. 4. As before, we reject the solutions with $s<0$ as unphysical. This leaves two physical solutions, one with $s \cong 0$ and the other with $s \cong 2$. We here record these roots in closed form for the sake of concreteness:

$$
\begin{aligned}
S= & \left(\frac{1}{2}(J+2)^{2}+\frac{1}{2} J^{2}\right. \\
& \left. \pm \frac{1}{2}\left\{\left[(J+2)^{2}-J^{2}\right]^{2}-4(g / 2 \pi)^{2}\left[4-(g / 2 \pi)^{2}\right]\right\}^{1 / 2}\right)^{1 / 2} \\
& -J .
\end{aligned}
$$

It should be mentioned that, using the methods of Ref. 4, one also discovers elementary amplitudes $\chi_{J}^{i}\left(q^{2}, \alpha\right)$ with certain integer values for the exponents $s(\alpha): s(\alpha)=5,6,7, \ldots$ The heuristic methods used in the present discussion are not sufficiently powerful to find these solutions easily. However, since these solutions fall off so quickly as $q^{2} \rightarrow \infty$, we will not need to be concerned with them.

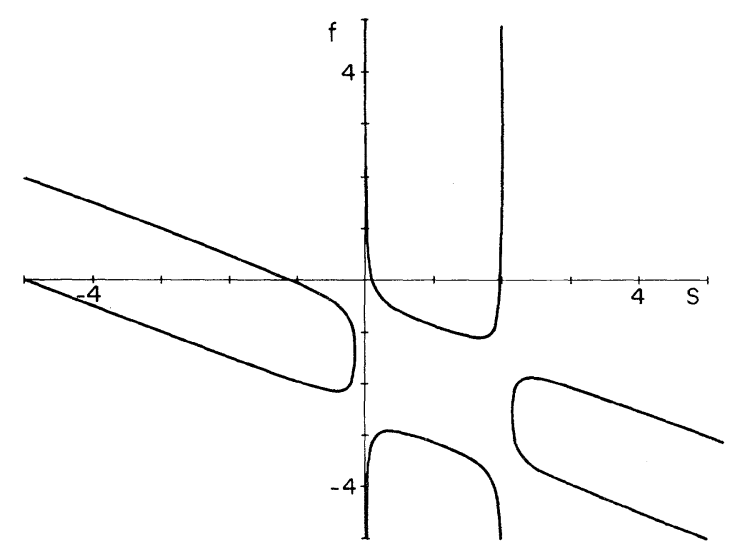

FIG. 4. The functions $f_{ \pm}(s)$. 
C. Behavior of the solutions in the complex $J$ plane

In the next section we will discuss the amplitudes $\chi_{J}^{i}\left(q^{2}\right)$ as analytic functions of $J$ and will look for their poles in the complex $J$ plane. As a preliminary step, let us notice that the explicit solutions for the large $-q^{2}$ coefficients $a_{J}^{i}$ are meromorphic functions of $J$, with poles at certain locations in the left-half $J$ plane.

The solution (3.8) with $\left(a_{J}^{1}, a_{J}^{2}\right) \neq 0$ has its leading (that is, rightmost) pole at

$$
J=-\frac{1}{2}-\frac{1}{2} s \text {. }
$$

Since $s \approx 1$ for small coupling constant, this pole is approximately at $J=-1$. Further poles and double poles are located at $J=-\frac{1}{2}-\frac{1}{2} s-N$ for $N$ $=1,2, \ldots$.

The solution (3.13) with $\left(a_{J}^{3}, a_{J}^{4}\right) \neq 0$ has its leading pole at

$$
J=-1-\frac{1}{2} s .
$$

In the case of a solution with $s \approx 0$, this pole lies near $J=-1$. The case of a solution with $s \approx 2$ will not be of much interest for us since the leading pole of such a solution $a_{J}^{i}$ lies further to the left, near $J=-2$. Further poles and double poles are located at $J=-1-\frac{1}{2} s-N$ for $N=1,2, \ldots$. It is also of interest to note that the pole at $J=-1-\frac{1}{2} s$ is a simple pole of $a_{J}^{4}$ but a double pole of $a_{J}^{3}$.

\section{POLES OF $\chi_{J}^{i}\left(q^{2}\right)$}

In the previous section we wrote a large $-q^{2}$ asymptotic expansion for the partial-wave ampli- tudes. We found the leading powers of $q^{2}$ that occur and, in addition, discovered poles near $J=-1$ in the coefficients $a_{J}^{i}$ of the leading terms of the large $-q^{2}$ expansion. Here we turn to the more difficult question of whether these poles persist in the full amplitudes $\chi_{J}^{i}\left(q^{2}\right)$.

\section{A. Rearrangement of the BS equation}

We begin with the full ladder-model Bethe-Salpeter equation (2.8):

$$
0=\sum_{l=1}^{4}\left(A_{J+1}^{j l} \chi_{J+1}^{l}+B_{J}^{j l} \chi_{J}^{l}+C_{J-1}^{j l} \chi_{J-1}^{l}\right) .
$$

This three-term recursion equation can be reduced to a two-term recursion equation because the matrices $A$ and $C$ are highly singular. We first multiply the equation by the vector $\xi_{(1)}^{j}$ $=(1,-1,0,0)$. This vector annihilates the matrix $C_{J-1}$, so we obtain an equation involving only $\chi_{J+1}$ and $\chi_{J}$. Similarly, multiplication by $\xi_{(2)}^{j}$ $=(J, J+2,0,0)$, which annihilates $A_{J+1}$, gives an equation involving only $\chi_{J}$ and $\chi_{J-1}$. Two more two-term equations follow by multiplication by $\xi_{(3)}^{j}=(0,0,1,0)$ and by $\xi_{(4)}^{j}=(0,0,0,1)$. As a result we obtain four equations, which can be written in matrix form as (after some relabling of the indices $J)$

$$
\sum_{i=1}^{4}\left(D_{J+1}^{j i} \chi_{J+1}^{i}+E_{J}^{j i} \chi_{j}^{i}\right)=0
$$

where

$$
\begin{aligned}
& D_{J+1}=\left[\begin{array}{cccc}
\frac{J+1}{J+2} M^{2} q & \frac{J+3}{J+2} M^{2} q & 2 M^{2} m & 0 \\
(J+1)\left(m^{2}+q^{2}-T_{J+1}\right) & (J+3)\left(m^{2}-q^{2}-T_{J+1}\right) & -(J+3) 2 m q & -\frac{(J+1)(J+3)}{J+2} 2 m q \\
0 & \frac{J+3}{J+2} m q & m^{2}-\frac{q^{2}}{J+2}-T_{J} & -\frac{(J+1)(J+3)}{(J+2)^{2}} q^{2} \\
\frac{J+1}{J+2} \frac{1}{2} M^{2} m & \frac{J+3}{J+2} \frac{1}{2} M^{2} m & -M^{2} q & 0
\end{array}\right], \\
& E_{J}=\left[\begin{array}{cccc}
m^{2}+q^{2}-T_{J} & -m^{2}+q^{2}+T_{J} & 2 m q & \frac{J}{J+1} 2 m q \\
-(J+2) q & (J+2) q & 0 & (J+2) 2 m \\
\frac{1}{2} m & -\frac{1}{2} m & 0 & q \\
0 & m q & -q^{2} & m^{2}+\frac{q^{2}}{J+1}-T_{J+1}
\end{array}\right] .
\end{aligned}
$$

We will want to use the Bethe-Salpeter equation to compute $\chi_{J}$, given $\chi_{J_{+1}}$. Thus we rewrite Eq. (4.1) in the form 


$$
\chi_{J}^{i}=\sum_{j=1}^{4} F_{J}^{i j} \chi_{J+1}^{j},
$$

where

$$
F_{J}=-E_{J}^{-1} D_{J+1} \text {. }
$$

The matrix $E_{J}^{-1}$, and thus $F_{J}$, can be calculated and written in closed form. This is a remarkable stroke of good fortune, since the matrix elements $E_{J}^{i j}$ contain the integral operators $T_{J}$ and $T_{J+1}$. Fortunately, however, $E_{J}{ }^{-1}$ turns out to contain positive powers of these operators only. ${ }^{12}$

When computing $E^{-1}$ and then $F=-E^{-1} D$ one must be careful of operator orderings. Let us write $T_{L}$ for the integral operator defined in Eq. (2.7) and $f(q)$ for the operator that multiplies by the function $f(q)$. Thus, for instance, with $f(q)=\left(q^{2}+m^{2}\right)^{-1}$ we write $T_{L}\left(q^{2}+m^{2}\right)^{-1}$ for the combined operator

$$
\left[T_{L} \frac{1}{q^{2}+m^{2}} h\right](q)=\frac{(g / 2 \pi)^{2}}{2(L+1)} \int d k \frac{k^{2}}{q} e^{-(L+1) \omega(k, q)} \frac{1}{k^{2}+m^{2}} h(k) .
$$

With this notation, the matrix elements of $F_{J}$ are

$$
\begin{aligned}
& F_{J}^{11}=\frac{(J+1)}{2(J+2)} \frac{1}{q}\left[q^{2}+m^{2}-M^{2}+\frac{1}{q^{2}+m^{2}} q T_{J} q-\frac{q^{2}+2 m^{2}}{q^{2}+m^{2}} T_{J+1}-\frac{1}{q^{2}+m^{2}}\left(q T_{J} q-m T_{J+1} m\right) \frac{1}{q^{2}+m^{2}} T_{J+1}\right], \\
& F_{J}^{12}=\frac{(J+3)}{2(J+2)} \frac{1}{q}\left[q^{2}-m^{2}+M^{2}+\frac{1}{q^{2}+m^{2}} q T_{J} q+\frac{q^{2}+2 m^{2}}{q^{2}+m^{2}} T_{J+1}+\frac{1}{q^{2}+m^{2}}\left(q T_{J} q-m T_{J+1} m\right) \frac{1}{q^{2}+m^{2}} T_{J+1}\right] \\
& F_{J}^{13}=\frac{m}{(J+2)}\left[-(J+3)-(J+2) \frac{1}{q^{2}+m^{2}} T_{J}+\frac{1}{q^{2}+m^{2}} \frac{1}{q} T_{J+1} q+(J+2) \frac{1}{q^{2}+m^{2}}\left(T_{J}+\frac{1}{q} T_{J+1} q\right) \frac{1}{q^{2}+m^{2}} T_{J}\right] \\
& F_{J}^{14}=\frac{(J+1)(J+3)}{(J+2)^{2}} m\left(-1+\frac{1}{q^{2}+m^{2}} \frac{1}{q} T_{J+1} q\right) \text {, } \\
& F_{J}^{21}=-\frac{(J+1)}{2(J+2)} \frac{1}{q}\left[q^{2}-m^{2}+M^{2}-\frac{1}{q^{2}+m^{2}} q T_{J} q-\frac{q^{2}-2 m^{2}}{q^{2}+m^{2}} T_{J+1}+\frac{1}{q^{2}+m^{2}}\left(q T_{J} q-m T_{J+1} m\right) \frac{1}{q^{2}+m^{2}} T_{J+1}\right], \\
& F_{J}^{22}=\frac{(J+3)}{2(J+2)} \frac{1}{q}\left[q^{2}+m^{2}-M^{2}-\frac{1}{q^{2}+m^{2}} q T_{J} q+\frac{q^{2}-2 m^{2}}{q^{2}+m^{2}} T_{J+1}-\frac{1}{q^{2}+m^{2}}\left(q T_{J} q-m T_{J+1} m\right) \frac{1}{q^{2}+m^{2}} T_{J+1}\right] \text {, } \\
& F_{J}^{23}=m\left[\frac{J+1}{J+2}-\frac{3}{q^{2}+m^{2}} T_{J}+\frac{1}{J+2} \frac{1}{q^{2}+m^{2}} \frac{1}{q} T_{J+1} q+\frac{1}{q^{2}+m^{2}}\left(T_{J}+\frac{1}{q} T_{J+1} q\right) \frac{1}{q^{2}+m^{2}} T_{J}\right] \\
& F_{J}^{24}=\frac{(J+1)(J+3)}{(J+2)^{2}} m\left(-1+\frac{1}{q^{2}+m^{2}} \frac{1}{q} T_{J+1} q\right) \text {, } \\
& F_{J}^{31}=-\frac{(J+1)}{2(J+2)} m\left[\frac{(J+2)}{(J+1)}-\frac{1}{q^{2}+m^{2}} \frac{1}{q} T_{J} q-\frac{(2 J+3)}{(J+1)} \frac{1}{q^{2}+m^{2}} T_{J+1}+\frac{1}{q^{2}+m^{2}}\left(\frac{1}{q} T_{J} q+T_{J+1}\right) \frac{1}{q^{2}+m^{2}} T_{J+1}\right] \\
& F_{J}^{32}=\frac{(J+3)}{2(J+2)} m\left[\frac{J}{(J+1)}-\frac{1}{q^{2}+m^{2}} \frac{1}{q} T_{J} q+\frac{(2 J+3)}{(J+1)} \frac{1}{q^{2}+m^{2}} T_{J+1}-\frac{1}{q^{2}+m^{2}}\left(\frac{1}{q} T_{J} q+T_{J+1}\right) \frac{1}{q^{2}+m^{2}} T_{J+1}\right] \text {, } \\
& F_{J}^{33}=\frac{1}{q}\left\{\frac{q^{2}}{(J+1)(J+2)}+m^{2}-M^{2}+\frac{1}{q^{2}+m^{2}}\left[\frac{q^{2}}{(J+1)}-2 m^{2}\right] T_{J}-\frac{1}{(J+2)} \frac{1}{q^{2}+m^{2}} q T_{J+1} q\right. \\
& \left.+\frac{1}{q^{2}+m^{2}}\left(m T_{J} m-q T_{J+1} q\right) \frac{1}{q^{2}+m^{2}} T_{J}\right\} \\
& F_{J}^{34}=\frac{(J+1)(J+3)}{(J+2)^{2}} \frac{1}{q}\left(\frac{q^{2}}{J+1}-\frac{1}{q^{2}+m^{2}} q T_{J+1} q\right) \\
& F_{J}^{41}=\frac{(J+1)}{2(J+2)} m\left(-1+\frac{1}{q^{2}+m^{2}} T_{J+1}\right) \text {, } \\
& F_{J}^{42}=\frac{(J+3)}{2(J+2)} m\left(-1+\frac{1}{q^{2}+m^{2}} T_{J+1}\right) \text {, } \\
& F_{J}^{43}=\frac{q}{(J+2)}+\frac{q}{q^{2}+m^{2}} T_{J} \\
& F_{J}^{44}=\frac{(J+1)(J+3)}{(J+2)^{2}} q \text {. }
\end{aligned}
$$




\section{B. Analytic continuation}

We have defined the partial-wave amplitudes $\chi_{J}^{i}$ for positive integer values of $J$ and have written the Bethe-Salpeter equation in a form in which it relates $\chi_{J}$ at $J=N$ to $\chi_{J}$ at $J=N+1: \quad \chi_{J}=F_{J} \chi_{J+1}$. However, the definition of the amplitudes $\chi_{J}$ can be extended to include complex $J$ by writing $\chi_{J}^{i}\left(q^{2}\right)$ as an integral over $(q+P)^{2}$ of the discontinuity of $\chi\left(q^{\nu}+P^{\nu}, q^{\nu}\right)$ in the mass $(q+P)^{2}$ of one leg. ${ }^{4}$ The amplitudes so defined are analytic functions of $J$ in the right half plane and have singularities in the left half plane at locations determined by the rate of fall off of the discontinuity of $\chi$ as $(q+P)^{2} \rightarrow \infty$.

One notices that the matrix $F_{J}$ in the BetheSalpeter equation, as written in Eq. (4.3), is an analytic function of $J$. Thus it is sensible to assume that the amplitudes $\chi_{J}^{i}$ continue to obey the equation $\chi_{J}=F_{J} \chi_{J+1}$ for complex $J$. In principle one should rederive this equation for complex $J$, using the definition of $\chi_{J}^{i}$ for complex $J$ given in Ref. 4. We have not carried out this program in detail, but we have verified the crucial step: Let $\psi\left(q^{\nu}, q^{\nu}+P^{\nu}\right)$ be a scalar wave function that satisfies a dispersion relation in the variable $(q+P)^{2}$ and define

$\psi^{\prime}\left(q^{\nu}, q^{\nu}+P^{\nu}\right)=g^{2} \int \frac{d^{4} k}{(2 \pi)^{4}} \frac{1}{(q-k)^{2}+\mu^{2}} \psi\left(k^{\nu}, k^{\nu}+P^{\nu}\right)$.

One can insert the dispersion integral for $\psi$ on the right-hand side of this equation and calculate the $(q+P)^{2}$ discontinuity of the output function $\psi^{\prime}$ as an integral over the discontinuity of the input function $\psi$. If one then projects the partial-wave amplitude $\psi_{J}^{\prime}$ for complex $J$ according to the definition of Ref. 4, one finds

$$
\psi_{J}^{\prime}\left(q^{2}\right)=\left[T_{J} \psi_{J}\right]\left(q^{2}\right),
$$

where $T_{J}$ is precisely the integral operator that appears the Bethe-Salpeter equation [see Eq. (2.7)]

Also, the results of the previous section make it evident that the analytically continued $\chi_{J}^{i}$ obeys the Bethe-Salpeter equation with the analytically continued $F_{J}$, at least in the $q^{2} \rightarrow \infty$ limit. Recall that we solved the large $-q^{2}$ version of the BS equation for integer $J$ and obtained an explicit solution involving $\Gamma$ functions for the amplitudes $a_{J}^{i}$. The solutions are meromorphic functions of $J$ and are bounded by powers of $|J|$ as $|J| \rightarrow \infty$ in the right-half $J$ plane. According to Carlson's theorem they are the unique functions of complex $J$ that continue the solutions for integer $J$ into the complex $J$ plane and share the good $|J|$ $\rightarrow \infty$ behavior of the amplitudes $\chi_{J}^{i}$ defined in Ref. 4. Finally we note that these analytically continued amplitudes do satisfy the large $-q^{2}$ BS equation (3.5) for complex as well as integer $J$ 。

\section{Sources of poles}

We wish to locate the leading (i.e., rightmost) singularities of $\chi_{J}^{i}$. As we will see, the singularities are poles and the leading poles occur near $J$ $=-1$. How do these poles arise? To see the basic mechanism, let us examine one term in the equation $\chi_{J}=F_{J} \chi_{J+1}$ :

$$
\chi_{J}^{1}=\frac{J+1}{2(J+2)} \frac{1}{q^{2}+m^{2}} T_{J} q \chi_{J+1}^{1}+\cdots .
$$

In more detail, this term is

$$
\begin{aligned}
\chi_{J}^{1}\left(q^{2}\right)= & \frac{(g / 2 \pi)^{2}}{4(J+2)} \frac{1}{q^{2}+m^{2}} \int_{0}^{\infty} d k \frac{k^{2}}{q} e^{-(J+1) \omega} k \chi_{J+1}^{1}(k) \\
& +\cdots
\end{aligned}
$$

where, we recall, $\cosh \omega=\left(q^{2}+k^{2}+\mu^{2}\right) / 2 q k$. There are four possible sources of poles evident in this equation.

First, $\chi_{J+1}^{1}$ could have a pole. If $\chi_{J+1}^{1}$ has a pole at $J+1=J_{\alpha}$, then $\chi_{J}^{1}$ will have a "daughter" pole at $J=J_{\alpha}-1$. However, let us look for the leading poles of $\chi_{J}^{i}$; that is, poles that are not daughters of other poles one unit to the right.

The second evident source of a pole is the factor $(J+2)^{-1}$. This possible pole is too far to the left to be of concern to us, but in other terms in $F_{J} \chi_{J+1}$ one finds factors $(J+1)^{-1}$ that could produce poles at $J=-1$. We will argue in Appendix B that these pole factors at $J=-1$ are canceled by zeros associated with the wave function at $J=0$. Thus there are no poles precisely at $J=-1$.

The third possible source of poles is the possible divergence of the integral at its $k \rightarrow 0$ end. We show in Appendix B that no such infared poles occur near $J=-1$.

Finally, we turn to a mechanism that does produce poles in $\chi_{J}^{i}$ : the $k$ integration can diverge at the ultraviolet end. For large $k, \exp [-(J+1) \omega]$ behaves like $(k / q)^{-(J+1)}$. In Sec. III we found how $\chi_{J+1}^{1}\left(k^{2}\right)$ behaves as $k \rightarrow \infty$; it is a sum of terms of the form

$$
\chi_{J+1}^{1}\left(k^{2}\right) \sim a_{J+1}^{1} k^{-3-s-(J+1)},
$$

where the smallest possible values of $s$ lie near $s=1$. Each such term in the asymptotic expansion of $\chi_{J+1}^{1}$ leads to a pole term in the integral on the right-hand side of Eq. (4.4):

$$
\begin{aligned}
\frac{(g / 2 \pi)^{2}}{4(J+2)} & \frac{1}{q^{2}+m^{2}} \int_{\Lambda}^{\infty} d k \frac{k^{2}}{q}\left(\frac{k}{q}\right)^{-(J+1)} k a_{J+1}^{1} k^{-3-s-(J+1)} \\
& =\frac{1}{2 J+s+1} \frac{q^{J}}{q^{2}+m^{2}} \frac{g^{2} /(2 \pi)^{2}}{4\left(J_{\alpha}+2\right)} a_{J_{\alpha}+1}^{1}+\text { analytic, }
\end{aligned}
$$

where $J_{\alpha}=-\frac{1}{2}-\frac{1}{2} s$ is the pole location. With $s \cong 1$, we have $J_{\alpha} \cong-1$.

Let us consider one more example of how poles 
arise from ultraviolet divergences in the BS equation. One term in $\chi_{J}^{3}\left(q^{2}\right)$ is

$$
\chi_{J}^{3}=-\frac{1}{q^{2}+m^{2}} T_{J+1} \frac{q}{q^{2}+m^{2}} T_{J} \chi_{J+1}^{3}+\cdots .
$$

The integ ral operator $I_{J} \equiv T_{J+1}\left[q /\left(q^{2}+m^{2}\right)\right] T_{J}$ has the kernel

$$
\begin{aligned}
I_{J}(q, k)= & \frac{(g / 2 \pi)^{4}}{4(J+1)(J+2)} \frac{k^{2}}{q} \\
& \times \int_{0}^{\infty} d P \frac{P^{2}}{P^{2}+m^{2}} e^{-(J+2) \omega(q, P)-(J+1) \omega(P, k)},
\end{aligned}
$$

$\omega\left(P_{1}, P_{2}\right)$ is defined in Eq. (2.6). The limiting behavior of this kernel as $k \rightarrow \infty$ with $q$ fixed as

$$
\begin{aligned}
I_{J}(q, k) \sim & \frac{(g / 2 \pi)^{4}}{4(J+1)(J+2)} q^{J+1} k^{1-J} \ln k \\
& +a(J, q) k^{1-J}+O\left(k^{-J}\right),
\end{aligned}
$$

where $a(J, q)$ is a not very simple function of $J$ and $q$. When $I_{J}$ is applied to a term in $\chi_{J+1}^{3}$ that behaves like $a_{J+1}^{3} k^{-3-s-(J+1)}$ for large $k$, the result has a leading singularity at $J=J_{\alpha} \equiv-1-\frac{1}{2} s$. Because of the $\ln k$ factor in $I_{J}(q, k)$, the singularity will consist of a double pole term plus a simple term:

$$
\begin{array}{lc}
I_{J} \chi_{J+1}^{3}=\frac{1}{(2 J+2+s)^{2}} \frac{(g / 2 \pi)^{4}}{4\left(J_{\alpha}+1\right)\left(J_{\alpha}+2\right)} q^{J_{\alpha}+1} & \begin{array}{c}
\operatorname{Res}_{J=J_{\alpha}} \chi_{J}^{4}\left(q^{2}\right)=0, \\
+\frac{1}{(2 J+2+s)} a\left(J_{\alpha}, q\right)+\text { analytic . }
\end{array} \\
C=\frac{(g / 2 \pi)^{2}}{32\left(J_{\alpha}+1\right)\left(J_{\alpha}+2\right)}\left\{\left[4 J_{\alpha}+4-(g / 2 \pi)^{2}\right] a_{J_{\alpha+1}}^{1}-\frac{J_{\alpha}+3}{J_{\alpha}+1}\left[4 J_{\alpha}+4+(g / 2 \pi)^{2}\right] a_{J_{\alpha^{+1}}}^{2}\right\} .
\end{array}
$$

As $q-0$, one would expect that $\chi_{J}^{i}\left(q^{2}\right)$ behaves like $q^{L}$ where, for integer $J, L$ is the number of $\hat{q}^{\mu}$ 's in the basis function $G_{J}^{i}$, that is, $L=J+\Delta_{i}$ where $\Delta_{1}=\Delta_{2}=0, \Delta_{3}=-1$, and $\Delta_{4}=+1$. Notice that the small- $q$ behavior of the residues $\chi_{J}^{i}\left(q^{2}\right)$ is $q^{J_{\alpha}}$ for $i=1,2$ and $q^{J_{\alpha}^{-1}}$ for $i=3$, as expected.

As $q \rightarrow \infty$, the asymptotic form of the residue of $\chi_{J}^{i}$ must be the same as the residue at $J=J_{\alpha}$ of the asymptotic form $a_{J}^{i} q^{-3-J-s}$ of $\chi_{J}^{i}$, as given in Sec. III, Eqs. (3.8) and (3.16). It is.

The full $q^{2}$ dependence of $\operatorname{Res} \chi_{J}^{i}$ can be most simply understood by defining the Bethe-Salpeter amplitude with one leg amputated:

$$
\hat{\chi}\left(q^{\nu}+P^{\nu}, q^{\nu}\right) \equiv \chi\left(q^{\nu}+P^{\nu}, q^{\nu}\right)(i q+m) .
$$

The partial-wave amplitudes of $\hat{\chi}$ are related to those of $\chi$ by $\hat{\chi}_{J}^{i}=G_{j}^{i j} \chi_{J}^{j}$, where $G^{i j}$ is a certain matrix given in Ref. 4. One finds that the residue of $\hat{\chi}_{J}^{i}$ at $J=J_{\alpha}$ has a pure power-law dependence on $q^{2}$ :

$$
\operatorname{Res}_{J=J_{\alpha}} \hat{\chi}_{J}^{i}\left(q^{2}\right)=\left(0,0, q^{J^{-1}} C, 0\right) .
$$

Thus the deviation at modest $q^{2}$ of $\operatorname{Res} \chi_{J}^{i}\left(q^{2}\right)$ from
It should by now be evident how the terms in the asymptotic expansion of $\chi_{J+1}^{j}\left(q^{2}\right)$ for $q^{2} \rightarrow \infty$ act to produce poles in $\chi_{J}^{i}\left(q^{2}\right)$, and how the locations of these poles and the associated Laurent coefficients can be calculated. We will spare the reader the details of this calculation, and simply state the results.

\section{Poles from asymptotic terms with $\left(a^{1}, a^{2}\right) \neq 0$}

Consider first one of the terms in the asymptotic expansion of $\chi_{J}^{i}\left(q^{2}\right)$ with the form $a_{J}^{i} q^{-3-J-s}$ with $a_{J}^{i}=\left(a_{J}^{1}, a_{J}^{2}, 0,0\right)$ as given by Eq. (3.8) and with $s$ $\approx 1$, as given by Eqs. (3.11) and (3.12). Each such term induces a simple pole in $\chi_{J}^{i}\left(q^{2}\right)$ near $J=-1$ at $J=J_{\alpha}=-\frac{1}{2}-\frac{1}{2} s$. The residue at this pole is

$$
\begin{aligned}
& \operatorname{Res}_{J=J_{\alpha}} \chi_{J}^{1}\left(q^{2}\right)=\operatorname{Res}_{J=J_{\alpha}} \chi_{J}^{2}\left(q^{2}\right)=\frac{q^{J_{\alpha}}}{q^{2}+m^{2}} C, \\
& \operatorname{Res}_{\substack{J=J_{\alpha}\\
}} \chi_{J}^{3}\left(q^{2}\right)=\frac{m q^{J_{\alpha^{-1}}}}{q^{2}+m^{2}} C, \\
& \operatorname{Res}_{J=J_{\alpha}} \chi_{J}^{4}\left(q^{2}\right)=0,
\end{aligned}
$$

where

its pure asymptotic power law dependence on $q^{2}$ is entirely due to the massive propagator on the $q^{\nu}$ leg of the wave function.

E. Poles from asymptotic terms with $\left(a^{3}, a^{4}\right) \neq 0$

Consider next one of the terms in the asymptotic expansion of $\chi_{J}^{i}\left(q^{2}\right)$ with the form $a_{J}^{i} q^{-3-J-s}$, with $a_{J}^{i}=\left(0,0, a_{J}^{3}, a_{J}^{4}\right)$ as given by Eq. (3.13) and with $s \approx 0$ as given by Eq. (3.15). Each such term induces a double pole ${ }^{13}$ near $J=-1$, at $J=J_{\alpha} \equiv-1$ $-\frac{1}{2} s$.

The coefficient of $\left(J+1+\frac{1}{2} s\right)^{-2}$ in the Laurent expansion of $\chi_{J}^{i}\left(q^{2}\right)$ about $J=J_{\alpha}$ is $\psi^{i}\left(q^{2}\right)$, where

$$
\begin{aligned}
\psi^{1}= & \psi^{2}=\frac{m q^{J_{\alpha}}}{q^{2}+m^{2}} \frac{(g / 2 \pi)^{4}\left(2 J_{\alpha}+3\right)}{16\left(J_{\alpha}+1\right)^{2}\left(J_{\alpha}+2\right)} a_{J^{+1}}^{3}, \\
\psi^{3}= & -q^{J_{\alpha}-1} \frac{\left(J_{\alpha}+1\right) q^{2}-\left(J_{\alpha}+2\right) m^{2}}{q^{2}+m^{2}} \frac{(g / 2 \pi)^{4}}{16\left(J_{\alpha}+1\right)^{2}\left(J_{\alpha}+2\right)} \\
& \times a_{J_{\alpha^{+1}}}^{3}, \\
\psi^{4}= & 0 .
\end{aligned}
$$

The $q^{-3-J-s}$ terms in $\chi_{J}^{i}\left(q^{2}\right)$ also produce single 
pole terms $r^{i} /\left(J+1+\frac{1}{2} s\right)$ in the Laurent expansion of $\chi_{J}^{i}$ about $J=-1-\frac{1}{2} s$. The residues $r^{i}$ are calculable, but we have not calculated them.

\section{F. Contribution from daughter asymptotic terms}

As we have seen, the $q^{-3-J-s}$ terms in $\chi_{J}^{i}\left(q^{2}\right)$ with $s \cong 0$ produce poles and double poles at $J$ $=-1-\frac{1}{2} s$. There is another source of (simple) pole terms at the same $J$. Recall from the discussion at the beginning of Sec. III that for each "parent" term $a_{J}^{i} q^{-3-J-s}$ in the asymptotic expansion of $\chi_{J}^{i}\left(q^{2}\right)$ there are "daughter" terms that fall off faster than the parent term by an integer number of powers of $q$ (except for some possible factors of $\ln q$ ). A complete analysis of the daughter terms is given in Ref. 2, and we rely on that analysis for the details of the present discussion.

Consider the amplitudes $\chi_{J}^{1}\left(q^{2}\right)$ and $\chi_{J}^{2}\left(q^{2}\right)$. The large $-q^{2}$ expansion of these amplitudes will contain terms $\hat{a}_{J}^{i} q^{-3-J-s-1}$ that are daughters of the $q^{-3-J-s}$ terms. ${ }^{14}$ According to our previous discussion of the effect of asymptotic terms with $\left(a^{1}, a^{2}\right) \neq 0$, such a daughter term will produce a simple pole at $J=-\frac{1}{2}-\frac{1}{2}(s+1)=-1-\frac{1}{2} s$. It is possible to calculate the residue of this pole term by first calculating the coefficients $\hat{a}_{J}^{i}$ of the daughter asymptotic terms, then using Eq. (4.5), but we have not done so.

No other daughter terms in the asymptotic expansion of $\chi_{J}^{i}\left(q^{2}\right)$ fall off slowly enough to produce a pole near $J=-1$.

\section{CONCLUSION}

The behavior of the pion wave function $\chi\left(q^{\nu}+p^{\nu}, q^{\nu}\right)_{\alpha \beta}$ as $(q+p)^{2} \rightarrow \infty$ with $q^{2}$ fixed is controlled by the rightmost singularities in the complex [O(4)] $J$ plane of the partial-wave amplitudes $\chi_{J}^{i}\left(q^{2}\right)$. These rightmost singularities play an important role, for instance, in determining the behavior of the pion electromagnetic form factor as $Q^{2} \rightarrow \infty .4$ The $J$-plane singularities of $\chi_{J}^{i}\left(q^{2}\right)$ can be studied in the limit $q^{2} \rightarrow \infty$ by using the requirement of conformal invariance at short distances: in the $q^{2}$ $\rightarrow \infty$ limit the leading singularities are poles near $J=-1$ whose locations are determined by the anomalous dimensions of certain operators. ${ }^{4}$

In order to apply this short-distance information to the pion form-factor problem, one is led to adopt a simple but unproven hypothesis ${ }^{3,4,5}$ :

(1) the rightmost singularities of $\chi_{J}^{i}\left(q^{2}\right)$ are poles;

(2) the positions of these poles are independent of $q^{2}$

(3) the residues of $\chi_{J}^{i}\left(q^{2}\right)$ at these poles have the same rates of fall-off as $q^{2} \rightarrow \infty$ as do the leading terms in the large $-q^{2}$ asymptotic expansion of $\chi_{J}^{i}\left(q^{2}\right)$.
On this hypothesis, the pole positions of $\chi_{J}^{i}\left(q^{2}\right)$ at modest $q^{2}$ are just those determined by the large $-q^{2}$ analysis.

The purpose of this paper has been to test this hypothesis in a model that is simple enough to permit calculation, namely, the ladder model with scalar gluon exchange. We have found that the hypothesis stated above has survived this laddermodel test.

(The short-distance analysis of Ref. 4 indicates that the $J$-plane poles should be simple poles. However, in a model like the ladder model in which the anomalous dimension of the quark field is zero, some of these poles are expected to coalesce into double poles. We do indeed find double poles as expected.)

In the calculation of the pion form factor using the large $-q^{2}$, conformal-invariant limit of the pion wave function, it is found $\mathrm{d}^{5,4}$ that the contribution arising from one set of poles of the wave function vanishes. The poles involved are those at $J=-\frac{1}{2}$ $-\frac{1}{2} s$ with $s \cong 1$, as given in Eqs. (3.11) and (3.12). (In the analysis of Ref. 4 , these poles are associated with odd chirality operators like $\bar{\psi} \gamma_{5} \psi$ in the operator-product expansion of two spinor fields.) The contribution to the pion form factor from such a pole is proportional to an integral of the form

$$
\begin{aligned}
\int_{0}^{\infty} d q^{2} & \left\{\left[\operatorname{Res}_{J=J_{\alpha}} \chi_{J}^{1}\left(q^{2}\right)-\operatorname{Res}_{J=J_{\alpha}} \chi_{J}^{2}\left(q^{2}\right)\right] A\left(q^{2}\right)\right. \\
& \left.+\operatorname{Res}_{\substack{J=J_{\alpha} \\
\operatorname{Res}_{J}}}\left(q^{2}\right) B\left(q^{2}\right)\right\} .
\end{aligned}
$$

When one replaces $\operatorname{Res} \chi_{J}^{i}\left(q^{2}\right)$ by its large- $q^{2}$ limit $r^{i} q^{-3-s-J_{\alpha}}$, one finds a cancellation because $r^{1}$ $-r^{2}=r^{4}=0$. One also finds that the exact residue will obey $\operatorname{Res} \chi^{1}-\operatorname{Res} \chi^{2}=\operatorname{Res} \chi^{4}=0$, thus giving an exact cancellation, provided that

$$
\operatorname{Res}_{J=J_{\alpha}} \chi_{J}^{i}\left(q^{2}\right)=H^{i j}\left(q^{2}\right) r^{j} q^{-3-s-J_{\alpha}} .
$$

Here $H^{i j}$ is a matrix of a certain form $\mathrm{m}^{4}$ such that multiplication of $\chi_{J}^{i}\left(q^{2}\right)$ by $H^{i j}$ corresponds to multiplication of $\chi\left(q^{\nu}+P^{\nu}, q^{\nu}\right)_{\alpha \beta}$, on the right by $\left[F\left(q^{2}\right) i \not q+G\left(q^{2}\right)\right]_{\beta^{\prime} \beta}$. (Thus only the leg of the wave function that carries modest momentum is modified.) We find that the residues of $\chi_{J}^{i}\left(q^{2}\right)$ at the poles in question are related to their large $-q^{2}$ limits in the required way in the ladder model. [We did not investigate the behavior of the residues at the other set of singularities of $\chi_{J}^{i}$, those at $J=-1-\frac{1}{2} s$ with $s \cong 0$ as given in Eq. (3.15).]

\section{ACKNOWLEDGMENT}

We wish to thank P. Menotti for helpful correspondence about his paper on conformal symmetry breaking. ${ }^{15}$ 


\section{APPENDIX A}

In this appendix we show briefly how the rightmost poles in the complex $J$ plane of the partialwave amplitudes $\chi_{J}^{i}\left(q^{2}\right)$ determine the large $-(q+P)^{2}$ behavior of the Bethe-Salpeter wave function $\chi\left(q^{\nu}+P^{\nu}, q^{\nu}\right)$. Many of the techniques used here are discussed more fully in Ref. 4.

Begin with the part of $\chi\left(q^{\nu}+P^{\nu}, q^{\nu}\right)$ that is proportional to $\gamma_{5}$ :

$$
\chi_{I}\left(q^{\nu}+P^{\nu}, q^{\nu}\right)=\sum_{J=0}^{\infty} \chi_{J}^{1}\left(q^{2}\right) i \gamma_{5}(-i M)^{J} U_{J}(z),
$$

where $z=\hat{q}^{4}=i \hat{q}^{0}=-i q^{\mu} P_{\mu} / M\left(q^{2}\right)^{1 / 2}$. To begin with, $z$ is real and $-1<z<1$. We wish to rewrite the sum in a form in which the limit $z \rightarrow \infty$ can be investigated.

First let us break up $U_{J}$ into two parts:

$$
U_{J}(z)=V_{J}(z)-V_{-J-2}(z),
$$

where

$$
V_{J}(z)=\frac{\left[z+\left(z^{2}-1\right)^{1 / 2}\right]^{J+1}}{2\left(z^{2}-1\right)^{1 / 2}} .
$$

The function $V_{J}(z)$ is defined with a cut along the real $-z$ axis from $z=-\infty$ to $z=+1$. In Eq. (A1) we choose to evaluate $V_{J}(z)$ and $V_{-J-2}(z)$ just below the cut. The crucial fact about $V_{J}(z)$ is its large$z$ behavior:

$$
V_{J}(z) \sim(2 z)^{J} \text { as } z \rightarrow \infty .
$$

Next, rewrite the sum involving $V_{J}(z)$ by means of a Sommerfeld-Watson transformation:

$$
\begin{aligned}
\chi_{I}=-\sum_{J=0}^{\infty} & \chi_{J}^{1}\left(q^{2}\right) i \gamma_{5}(-i M)^{J} V_{-J-2}(z) \\
& +\frac{1}{2} i \int d J \chi_{J}^{1}\left(q^{2}\right) i \gamma_{5} \frac{e^{i \pi J / 2}}{\sin \pi J} M^{J} V_{J}(z) .
\end{aligned}
$$

Initially, the integration contour circles the positive real- $J$ axis from $J=\infty-i \epsilon$. However, we have arranged our conventions so that the integrand falls off exponentially as $|J| \rightarrow \infty$. Thus the contour can be unfolded so that it runs just to the left of the imaginary $-J$ axis from $-\epsilon-i \infty$ to $-\epsilon+i \infty$.

At this point we can let $z$ be large and ask what happens as $z \rightarrow \infty$. Thus the large- $z$ behavior of the integral can be investigated by moving the integration contour to the left. When the contour has been moved past a pole in $\chi_{J}^{1}$ at, say, $J=J_{\alpha}$, a contribution

$$
\chi_{I}^{(\alpha)}=-i \gamma_{5}\left[\operatorname{Res}_{J=J_{\alpha}} \chi_{J}^{1}\left(q^{2}\right)\right] \frac{\pi e^{i \pi J_{\alpha} / 2} M^{J_{\alpha}}}{\sin \pi J_{\alpha}} V_{J_{\alpha}}\left(\frac{q \cdot P}{i M q}\right)
$$

poles yield the leading terms in the $(q+P)^{2} \rightarrow \infty$ limit.

One must, of course, worry about the "sum" term in (A5) and also the terms that arise when the integral is moved past a pole of $1 / \sin \pi J$ at $J=-1,-2, \ldots$ These terms cancel against one another provided

$$
M^{-N-2} \chi_{-N-2}^{1}\left(q^{2}\right)=(-1)^{N} M^{N} \chi_{N}^{1}\left(q^{2}\right)
$$

for $N=-1,0,1,2, \ldots$ These identities are called the Lorentz symmetry conditions for $\chi_{J}^{1}$. They are discussed in more detail in Ref. 4. As we see here they are the translation into the language of partial-wave amplitudes of the statement that the asymptotic expansion of $\chi_{I}\left(q^{\nu}+P^{\nu}, q^{\nu}\right)$ does not contain terms that fall off like $\left[(q+P)^{2}\right]^{-N-2}$.

In a realistic model in which all fields have anomalous dimensions, one would not expect $\left[(q+P)^{2}\right]^{-N-2}$ terms. Thus one would expect (A7) to hold for all $N$. In the ladder model one can have some doubt ${ }^{16}$ about the validity of these relations for all $N$. We prove the validity of some of the relations in the ladder model in Appendix B.

We have seen how $J$-plane poles are related to large- $(q+P)^{2}$ behavior in the part of the wave function proportional to $\gamma_{5}$. One can treat the other parts of the wave functions in an exactly analogous manner by defining invariant amplitudes $F_{1}, F_{2}$, $F_{3}, F_{4}$ as in Ref. 4:

$$
\chi\left(q^{\nu}+P^{\nu}, q^{\nu}\right)=i \gamma_{5} F_{1}+i[\not \supset, \not q] \gamma_{5} F_{2}+\not P \gamma_{5} F_{3}+\not q \gamma_{5} F_{4} .
$$

Each $F_{i}\left(q \cdot P, q^{2}\right)$ can be expanded in partial waves $f_{J}^{i}\left(q^{2}\right)$ :

$$
F_{i}=\sum_{J=0}^{\infty}(-i)^{J} f_{J}^{i}\left(q^{2}\right) U_{J}(z) .
$$

As before, the poles of $f_{J}^{i}$ determine the large $-q \cdot P$ behavior of $F_{i}$. The poles in the $f_{J}^{i}$ can be determined from the poles in the $\chi_{J}^{i}$ by using ${ }^{4}$

$$
\begin{aligned}
& \chi_{J}^{1}=M^{-J} f_{J}^{1}, \\
& \chi_{J}^{2}=-q M^{1-J}\left(\frac{J}{J+2} f_{J+1}^{2}+f_{J-1}^{2}\right), \\
& \chi_{J}^{3}=-\frac{1}{2} M^{-J}\left(M f_{J-1}^{3}-\frac{J}{J+1} q f_{J}^{4}\right), \\
& \chi_{J}^{4}=-\frac{1}{2} M^{-J}\left(q f_{J}^{4}+\frac{J+1}{J+2} M f_{J+1}^{3}\right) .
\end{aligned}
$$

\section{APPENDIX B}

In Sec. IV we found that $\chi_{J}^{i}\left(q^{2}\right)$ has poles on the real- $J$ axis near $J=-1$. These poles arise when the integral operators $T_{J}$ in the BS equation are applied to the wave functions: for certain values of $J$ the integrations diverge at their ultraviolet 
ends. We mentioned the possibility of divergences of the integrals at their infrared ends and mentioned the explicit factors of $1 /(J+1)$ that occur here and there in the Bethe-Salpeter equation. But we claimed that neither of these mechanisms produced poles of $\chi_{r}^{i}\left(q^{2}\right)$ near or to the right of $J=-1$. In this appendix we verify that claim.

First let us consider the possibility of a divergence at the $k \rightarrow 0$ end of one of the integrals in the BS equation. We first need to know how the wave functions $\chi_{s}^{i}\left(q^{2}\right)$ behave as $q^{2} \rightarrow 0$. One naturally expects that

$$
\chi_{J}^{i}\left(q^{2}\right) \sim C_{J}^{i} q^{J+\Delta} i
$$

as $q^{2} \rightarrow 0$, where $\Delta_{1}=\Delta_{2}=0, \Delta_{3}=-1$, and $\Delta_{4}=+1$. (For integer $J, J+\Delta_{i}$ is the number of factors of $q^{\mu}$ that occur in the basis function $G_{J}^{i}$ ) This hypothesis can easily be checked by inserting it on the right-hand side of the Bethe-Salpeter equation (4.2) and verifying that the same result is reproduced on the left-hand side.

Let us now use this result in a typical integral from the BS equation:

$$
\begin{aligned}
& {\left[T_{J} q \chi_{J+1}^{1}\right]\left(q^{2}\right)} \\
& \qquad=\frac{(g / 2 \pi)^{2}}{2(J+1)} \int_{0}^{\infty} d k \frac{k^{2}}{q} e^{-(J+1) \omega(\alpha, k)} k \chi_{J+1}^{1}\left(k^{2}\right) .
\end{aligned}
$$

The factor $\exp [-(J+1) \omega]$ behaves like $(k / q)^{J+1}$ as $k \rightarrow 0$ and $\chi_{J+1}^{1}\left(k^{2}\right)$ behaves like $k^{J+1}$. Thus the integral has a possible pole at $J=-3$ arising from an infrared divergence of the integration. When one examines all of the integrals in the BS equation (4.2) in this manner, one finds that the rightmost possible infrared poles are at $J=-2$. Thus these possible poles are not of concern to us.

(We suspect that the residues of the possible infrared poles vanish, but we have not pursued this question.)

There is one final source of poles near $J=-1$ that we must dispose of. The integral operator $T_{d}$, which occurs in several places in the Bethe-Salpeter equation (4.2), contains an explicit factor of $(J+1)^{-1}$ in its definition, Eq. (2.7). In addition, the BS equation contains a few more explicit factors of $(J+1)^{-1}$. We will show here that these factors do not produce poles in $\chi_{J}^{1}$ at $J=-1$.

Consider, for example, the term $F_{J}^{12} \chi_{J+1}^{2}$ that occurs in the BS equation (4.2) for $\chi_{J}^{1}$. According to Eq. (4.3), $F_{J}^{12}$ contains the operator $T_{J}$ and thus has superficially a pole at $J=-1$. However, $\chi_{J+1}^{2}$ must have a zero at $J+1=0$. To see this, con- sider the original form (2.8) of the BS equation for the partial-wave amplitudes. When one sets $J=1$ in this equation, one notices that it contains terms $C_{0}^{j 2} \chi_{0}^{2}$. However, there is no basis function $G_{0}^{2}$. For convenience in deriving Eq. (2.8) we have included a purely fictional term $G_{0}^{2} \chi_{0}^{2}$ in the $\mathrm{O}(4)$ expansion of $\chi(q+P, q)$, with the understanding that $\chi_{0}^{2}$ is to be zero by definition.

After deriving Eq. (2.8) for $J=0,1,2, \ldots$ we assume that the analytically continued amplitudes $\chi_{J}^{i}$ (defined as in Ref. 4) obeys the same equation for complex $J$. (This assumption was discussed in Sec.IV.) On this assumption the analytic function $\chi_{J}^{2}$ must have a zero at $J=0$ in order that the analytically continued BS equation match the integer $-J$ equation at $J=1$. Using this method one finds

$$
\begin{aligned}
& \chi_{0}^{2}=0, \\
& \chi_{0}^{3}=0 \\
& \chi_{-1}^{1}-\chi_{-1}^{2}-(2 m / q) \chi_{-1}^{4}=0 .
\end{aligned}
$$

The conditions (B2) help to cancel factors of $(J+1)^{-1}$, but they are not sufficient. Consider, for example, the following term in the BS equation (4.2) for $\chi_{J}^{1}$ :

$$
\frac{m}{q^{2}+m^{2}} T_{J} \frac{1}{q^{2}+m^{2}} T_{J} \chi_{J+1}^{3} .
$$

Each operator $T_{J}$ contains a factor $(J+1)^{-1}$, but we have no reason to believe that $\chi_{J+1}^{3}\left(k^{2}\right)$ has more than a first-order zero at $J+1=0$.

In order to find the extra factor of zero needed in Eq. (B3) we now return to a significant technical point that was omitted from the discussion of the large $-q^{2}$ behavior of $\chi_{J}^{1}\left(q^{2}\right)$ in Sec。III. Recall that the Bethe-Salpeter equation (2.8) contains expressions like $T_{J-1} \chi_{J}^{3}$ or, more generally,

$$
\begin{aligned}
{\left[T_{J+\Delta_{i}} \chi_{J}^{i}\right] } & \left(q^{2}\right) \\
& \equiv \frac{(g / 2 \pi)^{2}}{2\left(J+\Delta_{i}+1\right)} \int_{0}^{\infty} d k \frac{k^{2}}{q} e^{-(J+\Delta+1) \omega(\alpha, k)} \chi_{J}^{i}\left(k^{2}\right),
\end{aligned}
$$

where $\Delta_{1}=\Delta_{2}=0, \Delta_{3}=-1$, and $\Delta_{4}=+1$. Suppose we wish to know how $T_{J+\Delta_{i}} \chi_{J}^{i}$ behaves as $q \rightarrow \infty$, given an input ansatz for the large- $k$ behavior of $\chi_{J}^{i}$. To do this we define the Mellin transform ${ }^{2}$ of $\chi_{J}^{i}$ :

$$
\tilde{\chi}_{J}^{i}(\delta)=\int_{0}^{\infty} d k k^{6+2} \chi_{J}^{i}(k)
$$

Then, by adding and subtracting a term on the right-hand side of (B4) we can write

$$
\left[T_{J+\Delta_{i}} \chi_{J}^{i}\right]\left(q^{2}\right)=\frac{(g / 2 \pi)^{2}}{2\left(J+\Delta_{i}+1\right)} q^{-(J+\Delta+2)} \tilde{\chi}_{J}^{i}\left(J+\Delta_{i}+1\right)+\frac{(g / 2 \pi)^{2}}{2\left(J+\Delta_{i}+1\right)} \int_{0}^{\infty} d k \frac{k^{2}}{q}\left\{e^{-\left(J+\Delta_{i}+1\right) \omega(q, k)}-\left(\frac{q}{k}\right)^{-\left(J+\Delta_{i}+1\right)}\right\} \chi_{J}^{i}\left(k^{2}\right) .
$$


As $q \rightarrow \infty$, the factor in braces in the integrand tends to

$$
\theta(k-q)\left[\left(\frac{k}{q}\right)^{-\left(J+\Delta_{i}+1\right)}-\left(\frac{q}{k}\right)^{-\left(J+\Delta_{i}+1\right)}\right] .
$$

Thus the asymptotic form of the integral in (B6) depends only on the asymptotic form of $\chi_{J}^{i}\left(k^{2}\right)$. If we insert $\chi_{J}^{i}\left(k^{2}\right) \sim a_{J}^{i} k^{-3-s-J}$ into the integral we obtain

$$
\frac{(g / 2 \pi)^{2}}{(J+\Delta+1)^{2}-(J+s)^{2}} q^{-1-s-J}
$$

as $q \rightarrow \infty$. This is the result recorded in Eq. (3.4).

There remains the term proportional to the Mellin-transformed wave function $\tilde{\chi}_{J}^{i}(\delta)=J+\Delta_{i}+1$. We demand that the Bethe-Salpeter equation be satisfied term by term in an asymptotic expansion for large $q^{2}$. But there is no other term in the BS equation that behaves like $q^{-(J+\Delta+2)}$. Thus consistency requires that

$$
\tilde{\chi}_{J}^{i}(\delta)=0 \text { at } \delta=J+\Delta_{i}+1 \text {. }
$$

(Our discussion here has been heuristic. A rigorous derivation valid for integer $J$ is given in Ref. 2.)

The result (B7) provides the extra factor of zero needed in the expression (B3). Looking at Eq. (B4) we note that $T_{J+\Delta_{i}} \chi_{J}^{i}$ contains an explicit factor $\left(J+\Delta_{i}+1\right)^{-1}$. However we see from the representation (B6) that this factor does not produce a pole at $J+\Delta_{i}+1=0$ because the first term in (B6) is identically zero and in the second term the pole is canceled by a zero of the integrand at $J+\Delta_{i}+1=0$. Thus in the expression (B3) the rightmost operator $T_{J}$ is not singular at $J=-1$ (after we have rewritten it and thrown away the Mellin-transform term) and the pole of the leftmost operator $T_{J}$ at $J=-1$ is canceled by the zero of $\chi_{J+1}^{3}$ at $J+1=0$.

The reader is now in a position to verify, using
Eqs. (B2) and (B7), that all of the factors $(J+1)^{-1}$ in the Bethe-Salpeter equation are canceled.

One can also ask whether the Lorentz symmetry conditions on the wave function are satisfied in the ladder model. These conditions are $\mathrm{ar}^{4}$

$$
\begin{aligned}
& M^{J} \chi_{J}^{1}-(-1)^{J} M^{-J-2} \chi_{-J-2}^{1}=0, \\
& (J+2) M^{J} \chi_{J}^{2}+J(-1)^{J} M^{-J-2} \chi_{-J-2}^{2}=0, \\
& (J+1) M^{J} \chi_{J}^{3}+J(-1)^{J} M^{-J-2} \chi_{-J-2}^{4}=0,
\end{aligned}
$$

for $J=0, \pm 1, \pm 2, \ldots$ The first few conditions are

$$
0=\chi_{0}^{2}=\chi_{0}^{3}=\chi_{-1}^{1}=\chi_{-1}^{2}=\chi_{-1}^{4} .
$$

We have already seen [Eq. (B2)] that $\chi_{0}^{2}, \chi_{0}^{3}$, and the combination $\chi_{-1}^{1}-\chi_{-1}^{2}-(2 m / q) \chi_{-1}^{4}$ vanish.

One can also show that $\chi_{-1}^{4}=0$. One uses the Bethe-Salpeter equation $\chi_{J}^{i}=F_{J}^{i j} \chi_{J+1}^{j}$ and applies the same techniques that were used above to show that $\chi_{J}^{i}$ has no poles at $J=-1$. Special care is needed with the term in $\chi_{J}^{4}$ proportional to $T_{J} \chi_{J+1}^{3}$. Although $\chi_{J+1}^{3}$ vanishes at $J=-1, T_{J}$ contains a factor $(J+1)^{-1}$ that would cancel this zero and give a finite value for $\chi_{-1}^{4}$ if $\chi_{J_{+1}}^{3}(k)$ were an arbitrary function of $k$. However, the Bethe-Salpeter equation supplies the extra dynamical information that the Mellin transform $\tilde{\chi}_{J+1}^{3}(\sigma)$ vanishes at $\sigma=J+1$, [See Eq. (B7)]. With this information one finds, using Eq. (B6), that $\chi_{-1}^{4}=0$. The information that $\tilde{\chi}_{J+1}^{3}(J+1)=0$ was missing in the perturbative calculation ${ }^{15}$ of Menotti; this accounts for his obtaining a $\left(Q^{2}\right)^{-1}$ term in the pion form factor.

One can easily check, using the results of Ref. 4 , that the Lorentz symmetry conditions derived here, namely $\chi_{0}^{2}=\chi_{0}^{3}=\chi_{-1}^{1}-\chi_{-1}^{2}=\chi_{-1}^{4}=0$ are sufficient to guarantee that the ladder-model pion form factor does not have a $\left(Q^{2}\right)^{-1}$ term in its asymptotic expansion for large $Q^{2}$. It remains an open question whether the remaining Lorentz symmetry conditions are valid in the ladder model.
*Work supported in part by the National Science Foundation under Grant No. MPS 75-22514 and in part by the U. S. Energy Research and Development Administration.

${ }^{1}$ The full kernel is an appropriate amputated four-point function, with dimension $-2-4 \gamma_{\psi}$, times two fermion propagators, each with dimension $-1+2 \gamma_{\psi}$, where $\gamma_{\psi}$ is the anomalous dimension of the Fermi field. Thus $d^{4} p$ times the kernel is exactly zero dimension.

${ }^{2}$ A. H. Guth and D. E. Soper, Phys. Rev. D 12, 1143 (1975). This analysis proceeds by taking a Mellin transform of the BS equation.

${ }^{3}$ C. G. Callan and D. J. Gross, Phys. Rev. D 11, 2905 (1975).

${ }^{4}$ M. L. Goldberger, D. E. Soper, and A. H. Guth, Phys.
Rev. D 14, 1117 (1976).

${ }^{5}$ P. Menotti, Phys. Rev. D 13, 1778 (1976).

${ }^{6}$ E. E. Salpeter and H. A. Bethe, Phys. Rev. 84, 1232 (1951); M. Gell-Mann and F. E. Low, ibid. 84,350 (1951).

${ }^{7}$ Briefly, we use $g_{00}=-1, g_{i i}=+1,\left\{\gamma^{\mu}, \gamma^{\nu}\right\}=2 g^{\mu \nu}$, and for any vector $V^{\mu}$ we define $V^{4}=i V^{0}$.

${ }^{8}$ G. C. Wick, Phys. Rev. 96, 1124 (1954). See Ref. 2 for a further explanation of the notation.

${ }^{9}$ Several useful identities for manipulating the traceless symmetric operation are given in Ref. 2 .

${ }^{10}$ See, for example, Handbook of Mathematical Functions, edited by M. Abramowitz and I. A. Stegun, National Bureau of Standards Applied Mathematics Series, No. 55 (U. S. G. P.O., Washington, D. C., 1964), Chap. 
22.

${ }^{11}$ The general form follows from $\mathrm{O}(4)$ invariance. To evaluate the coefficient, multiply by $\hat{q}_{\mu_{1}} \cdots \hat{q}_{\mu_{L}}$ to obtain $2^{-L} U_{L}(\hat{q} \cdot \hat{k})$ on the left-hand side and $2^{-L_{L}} U_{L}(1)$ on the right-hand side.

${ }^{12}$ This good fortune can be traced to the fact that the formal matrix determinant of $E$ does not contain the operators $T_{L}$.

${ }^{13}$ The occurrence of a leading double pole in $a_{J}^{i}$ seems to be an artifact of the ladder model. The operatorproduct expansion of Ref. 4 [see especially Eq. (4.12) of Ref. 4] indicates that $a_{J}^{3}$ contains a product of two pole factors whose pole locations differ by the anomalous dimension $\gamma_{\psi}$ of the quark field $\psi(x)$. In the ladder model $\gamma_{\psi}=0$.

${ }^{14}$ See Ref. 2. No factor of $\ln q$ is associated with this term.

${ }^{15}$ P. Menotti, Phys. Rev. D (to be published).

${ }^{16}$ The possible doubt arises from the fact that the large$q^{2}$ expansion of $\chi_{J}^{i}\left(q^{2}\right)$ contains some nonleading terms that fall off like integer powers of $q^{2}$.

${ }^{17}$ This is a change of notation from Ref. 4 , where for physical values of $P^{\nu}$ we used the notation $\hat{P}^{\nu} \equiv P^{\nu} /$ $\left(P^{2}\right)^{1 / 2}=P^{\nu} /(-i M)=(0,0,0,-1)$. 